\title{
Patient-made Long Covid empowers the patient voice
}

\author{
Elisa Perego ${ }^{1}$ \\ ${ }^{1}$ Affiliation not available
}

December 21, 2021

\begin{abstract}
Successful, patient-led advocacy and research in Long Covid is contributing to change our understanding of SARS-CoV-2 infection, viral-onset diseases, and knowledge building in medicine and beyond. It is my hope that patient-centred expertise will be further incorporated within the biomedical community. This would contribute to critical changes in medical awareness of chronic diseases and patient care.
\end{abstract}

Successful, patient-driven Long Covid advocacy and research is, I argue, a huge opportunity to strengthening patient voices across different disciplines, patient communities, and biomedical fields.

SARS-CoV-2 is now recognized as a virus associated with high mortality and morbidity [1]. As of 31 October 2021, Covid-19 has caused 5 million official deaths worldwide. Many more people, however, never fully recovered from the initial infection and now suffer from persistent ill health, including organ impairment [2], neurological damage [3], endothelial dysfunction [4] and immune system dysfunction [5].

Long Covid has, therefore, emerged as a major clinical challenge across the pandemic [6]. In the UK alone, over one million people are reported to suffer from long-term symptoms and sequelae following SARS-CoV-2 infection. This is shown by data from the ONS (Office for National Statistics) [7] and the REACT-2 study [8]. Data from other countries remain more sparse because of less refined collection methods, but are of severe concern. A paper published in the New England Journal of Medicine in August 2021 suggested that 15 million people might have Long Covid in the US [9].

Already in early 2020, those with Long Covid and from other chronic illness communities, started to raise the alarm on the catastrophic effects of unmitigated SARS-CoV-2 spread, and the enormous disease burden to come [10]. In an Open Letter published in September 2020, my co-authors and I defined the costs of Long Covid as "unfathomable" [11]. In a New York Times Opinion from March 2021, Lowenstein and Davis described the Covid-19 pandemic as "one of the most disabling events in modern history" [12].

Long Covid and cognate term Long Haul Covid were coined and brought to wide attention in the first pandemic months by a grassroots, international, patient-led movement of people, to which I participated actively [13].

Long Covid was identified, named, and defined collectively by patients. Intense mobilization via social and other media successfully challenged early official guidelines, which posed recovery from Covid-19 within $2-6$ weeks from symptom onset.

The definition of Long Covid as a disease entity took place via different means. Crucial was vast engagement of patients with public discussion of prolonged symptoms via patient-made hashtags. Equally important was patient engagement with the growing scientific literature on Covid-19, and the sharing of one's objective 
clinical data across different platforms (e.g. hypoxemia, abnormal cardiac function and raised ddmer levels beyond the acute phase of Covid-19). Innovative forms of data collection and analysis were developed in support groups and via social media. Healthcare professionals and scientists with Long Covid participated actively in knowledge production. Interdisciplinary participation of patient researchers with backgrounds in linguistics, history etc. was crucial to building a new language to define a disease.

The term Long Covid is now widely used in the scientific literature, as well as by policy makers, the media, and major health bodies [14].

As a patient-made term, and movement, that gained wide recognition in just a few months, Long Covid has prompted significant changes in how scientific knowledge is built [15]. Such changes will be hopefully durable. Those with Long Covid have produced ground-breaking research, advocacy, and science communication. This often happened in advance of conventional medicine and contributed to change how Covid-19 is understood and communicated to the public [16].

As physician-scientist Z. Al-Aly wrote in a Guardian Opinion in October 2021 "In a short few months [these patients-advocates-researchers] created a formidable patient-led advocacy and research movement that changed the arc of medical history" [17].

The experience of Long Covid is, therefore, crucial in medicine because it highlights the power and knowledge that lie within a patient community. Those with Long Covid must be recognized as experts on SARS-CoV-2 infection symptoms and sequelae in their own right.

Long Covid helped to widen the range of actors who are involved in public health decision-making, as well as in the matter of defining diseases, symptoms, and concepts of disability. For example, patient-led advocacy was instrumental to the landmark World Health Organization meeting of 21 August 2020, which publicly recognized Long Covid [18]. Patient researchers have also contributed to writing or reviewing guidelines at the national and international level.

I hope, therefore, that the experience of Long Covid will further galvanize different patient communities to strongly engage in grassroots activism and policy making. This is a delicate point, however, that need to be addressed with sensitivity. The onus of advocacy cannot be on patients alone, especially in a pandemic world with faltering economies and healthcare systems. Policy makers and new advocates, or allies, need to be aware of the long-term struggles, and successes, of the disability community across space and time.

At the same time, patient expertise must be incorporated into decision-making on matters of health and healthcare at the highest level.

Long Covid is contributing to a paradigm shift in our awareness and understanding of viral-onset diseases. It is encouraging that new awareness on the long-term effects of viral and other infections is bringing more visibility to (post) infection conditions. This must result into targeted funding, high-quality biomedical research, diagnostic, treatment, and support. I highlight again conditions such as myalgic encephalomyelitis/ chronic fatigue syndrome, dysautonomia, post-sepsis syndrome, and post-infectious vasculitis. These remain poorly researched, communicated to stakeholders, and clinically treated. Virus-induced lung diseases are another widespread clinical challenge [19] which deserve further consideration as long-term sequelae of Covid19.

I welcome growing awareness of other poorly researched conditions, such as fibromyalgia and connective tissue disorders. Notable is raising interest in their possible autoimmune and/or (post) infection component, which remain to be further characterized [20]. I also encourage engagement with expertise on autoimmune diseases, such as lupus (SLE), and thrombotic or inflammatory conditions. This is because of mounting evidence of autoimmunity, coagulopathy and inflammatory processes in Covid-19 [21] and Long Covid [22].

We need to address (post) infection diseases as complex clinical entities, which would benefit from an interdisciplinary biomedical approach for optimal understanding: for example, from cardiology, haematology, immunology etc. Knowledge building by patients and patient researchers would be crucial, too. Strategies 
that have proven key to grassroots Long Covid advocacy in the digital era could be further explored and expanded.

Some of the conditions I mentioned above, such as fibromyalgia, are more commonly reported in women. Sadly, this makes gender-based discrimination a likely factor in the lack of treatment and recognition [23] with a potential impact on patient-led research, too. Men and non-binary individuals may face, however, their own barriers to care, especially for diseases often characterized as more widespread in women.

The Long Covid movement has been largely built on leadership from women and people from marginalized and minoritazed communities, including disabled people/ people with disabilities. This was especially the case for the first wave in 2020. This contribution to knowledge has been ground-breaking and must be recognized as such.

I strongly recommend, thus, for the patient-led research model that has gained wider recognition in Long Covid [24], to become the norm for both Covid-19 and other diseases. It is my hope that patient-centred expertise will be increasingly incorporated within the biomedical community. This would contribute to critical changes in medical awareness of chronic diseases and patient care.

\title{
Author
}

\author{
Dr Elisa Perego (MA, PhD) \\ University College London (UK) \\ e.perego@ucl.ac.uk
}

\section{Acknowledgements}

I want to thank the Long Covid and disability community for constant engagement and thought-provoking debate, especially on Twitter. Barbara Melville-Jóhannesson (Creative Informatics, University of Edinburgh) has been an important source of encouragement as regards an early draft of this piece. I addressed various points raised in this paper at conferences such as the European Sepsis Alliance Annual Meeting and the World Sepsis Congress in 2021.

\section{Competing interests}

None

\section{Bibliography}

[1] Al-Aly et al 2021

https://www.nature.com/articles/s41586-021-03553-9

[2] Dennis et al 2021

https://bmjopen.bmj.com/content/11/3/e048391

[3] Morand et al 2021

https://link.springer.com/article/10.1007/s00259-021-05528-4

[4] Fogarty et al 2021

https://onlinelibrary.wiley.com/doi/abs/10.1111/jth.15490

[5] Buonsenso et al 2021

https://www.thelancet.com/journals/lanchi/article/PIIS2352-4642(21)00196-6/fulltext?dgcid=hubspot_email_newsletter_lancetcovid21\&utm_campaign=lancetcovid21\&utm_medium=email\&_hsmi=147137916\&_hsenc=p2ANqtz-bavMfmygYSwF3w4JkcRJVg5npAox1UXtJwl2VR5OBUY7CUYRDCcDQ_y9eyyiQi64MBShSv3MJcL0ZLuGbi6de8LNYw\&utm_content=147113269\&utm_source=hs_email 
[6] Sivan et al 2021

https://www.bmj.com/content/373/bmj.n853

[7] Official for National Statistics ONS

https://www.ons.gov.uk/peoplepopulationandcommunity/healthandsocialcare/conditionsanddiseases/bulletins/prevalenceof

[8] Whitaker et al 2021

https://spiral.imperial.ac.uk/handle/10044/1/89844

[9] Phillips and Williams 2021

https://www.nejm.org/doi/full/10.1056/NEJMp2109285

[10] Callard and Perego 2021

https://www.sciencedirect.com/science/article/pii/S0277953620306456

[11] Perego et al 2020a

https://wellcomeopenresearch.org/articles/5-224

[12] Lowenstein and Davis 2021

https://www.nytimes.com/2021/03/17/opinion/long-covid.html

[13] Perego et al 2020b

https://blogs.bmj.com/bmj/2020/10/01/why-we-need-to-keep-using-the-patient-made-term-long-covid/

[14] Floridia et al 2021

http://www.nbst.it/1081-long-covid-rapporto-iss-per-identificare-pazienti-comprendere-variabilit\%C3\%A0dei-sintomi-favorire-assistenza-multidisciplinare.html

[15] Perego and Callard 2021

https://osf.io/preprints/socarxiv/n8yp6/

[16] Yong 2020

https://www.theatlantic.com/health/archive/2020/08/long-haulers-covid-19-recognition-support-groupssymptoms/615382/

[17] Al-Aly 2021 The WHO is letting down long Covid patients. Guardian Opinion

https://www.theguardian.com/commentisfree/2021/oct/17/who-long-covid-patients

[18] Lokugamage et al 2020

https://blogs.bmj.com/bmj/2020/09/03/we-have-heard-your-message-about-long-covid-and-we-will-actsays-who/

[19] Garcia et al 2019

https://ajp.amjpathol.org/article/S0002-9440(19)30861-2/fulltext

[20] Goebel et al 2021

https://www.jci.org/articles/view/144201

[21] Wang et al 2021

https://www.nature.com/articles/s41586-021-03631-y 
[22] Pretorius et al 2021

https://cardiab.biomedcentral.com/articles/10.1186/s12933-021-01359-7

[23] Alwan 2021

https://www.nature.com/articles/s43856-021-00016-0

[24] Davis et al 2021

https://www.thelancet.com/journals/eclinm/article/PIIS2589-5370(21)00299-6/fulltext 